\title{
A New Theorem on The Existence of Positive Solutions of Singular Initial-Value Problem for Second Order Differential Equations
}

\author{
Afgan Aslanov ${ }^{1 *}$
}

\section{Abstract}

We proved a new theorem on the existence of positive solutions to initial-value problems for second-order nonlinear singular differential equations. The existence of solutions is proven under considerably weaker than previously known conditions.

Keywords: Second order equations, Existence, Emden-Fowler equation

2010 AMS: 34A12

${ }^{1}$ Computer Engineering Department, Istanbul Esenyurt University, Istanbul, Turkey

*Corresponding author: afganaslanov@yahoo.com

Received: 30 August 2018, Accepted: 17 November 2018, Available online: 22 March 2019

\section{Introduction}

We consider the problem

$$
\begin{aligned}
\left(p y^{\prime}\right)^{\prime}+p q g(y) & =0, t \in[0, T] \\
y(0) & =a>0, \\
\lim _{t \rightarrow 0+} p(t) y^{\prime}(t) & =0
\end{aligned}
$$

and

$$
\begin{aligned}
\left(p y^{\prime}\right)^{\prime}+p q g(y) & =0, t \in[0, T] \\
y(0) & =a>0, \\
y^{\prime}(0) & =0,
\end{aligned}
$$

where $0<T<\infty, p \geq 0, q \geq 0$ and $g:[0, \infty) \rightarrow[0, \infty)$.

Agarwal and O'Regan [1] established the existence theorems for the positive solution of the problem (1.1) and (1.2):

Theorem 1.1. [1] Suppose the following conditions are satisfied

$$
p \in C[0, T) \cap C^{1}(0, T) \text { with } p>0 \text { on }(0, T)
$$




$$
q \in L_{p}^{1}\left[0, t^{*}\right] \text { for any } t^{*} \in(0, T) \text { with } q>0 \text { on }(0, T)
$$

where $L_{r}^{1}[0, a]$ is the space of functions $u(t)$ with $\int_{0}^{a}|u(t)| r(t) d t<\infty$,

$$
\int_{0}^{t^{*}} \frac{1}{p(s)} \int_{0}^{s} p(x) q(x) d x d s<\infty \text { for any } t^{*} \in(0, T)
$$

and

$$
g:[0, \infty) \rightarrow[0, \infty) \text { is continuous, nondecreasing on }[0, \infty) \text { and } g(u)>0 \text { for } u>0
$$

Let

$$
H(z)=\int_{z}^{a} \frac{d x}{g(x)} \text { for } 0<z \leq a
$$

and assume

$$
\int_{0}^{t^{*}} \frac{1}{p(s)} \int_{0}^{s} p(x) q(x) \tau(x) d x d s<a \text { for any } t^{*} \in(0, T),
$$

here

$$
\tau(x)=g\left(H^{-1}\left(\int_{0}^{x} \frac{1}{p(w)} \int_{0}^{w} p(z) q(z) d z d w\right)\right) .
$$

Then (1.1) has a solution $y \in C[0, T)$ with $p y^{\prime} \in C[0, T),\left(p y^{\prime}\right)^{\prime} \in L_{p q}^{1}(0, T)$ and $0<y(t) \leq a$ for $t \in[0, T)$. In addition if either

$$
p(0) \neq 0
$$

or

$$
p(0)=0 \text { and } \lim _{t \rightarrow 0+} \frac{p(t) q(t)}{p^{\prime}(t)}=0
$$

holds, then $y$ is a solution of (1.2).

The condition (1.7) in connection with the definition of the function $\tau(x)$, makes this theorem difficult for an application. In [2] we proved more easy and applicable theorem:

Theorem 1.2. Suppose (1.3)-(1.5) hold. In addition, we assume

$$
\int_{0}^{t^{*}} \frac{1}{p(s)} \int_{0}^{s} p(x) q(x) g(a) d x d s<a
$$

for any $t^{*} \in\left(0, T_{0}\right)$. Then

a) (1.1) has a solution $y \in C\left[0, T_{0}\right)$ with $p y^{\prime} \in C\left[0, T_{0}\right),\left(p y^{\prime}\right)^{\prime} \in L_{p q}^{1}\left(0, T_{0}\right)$ and $0<y(t) \leq$ a for $t \in\left[0, T_{0}\right)$.

b) If $\int_{T_{0}}^{T_{1}} \frac{1}{p(s)} \int_{0}^{s} p(x) q(x) g\left(T_{0}\right) d s<y\left(T_{0}\right)$, and conditions (1.3)-(1.6) satisfied then solution can be extended into the interval $\left[0, T_{1}\right)$.

In this paper we generalized the Theorem 1.2. 


\section{Main result}

Theorem 2.1. Suppose the following conditions are satisfied

$$
\begin{aligned}
& p \in C[0, T) \cap C^{1}(0, T) \text { with } p>0 \text { on }(0, T], \\
& q \geq 0, \\
& \int_{0}^{t^{*}} \frac{1}{p(s)} \int_{0}^{s} p(x) q(x) d x d s<\infty \text { for any } t^{*} \in(0, T], \\
& g:[0, \infty) \rightarrow[0, \infty) \text { is continuous, nondecreasing on }[0, \infty),
\end{aligned}
$$

and assume

$$
\begin{aligned}
\int_{0}^{t} \frac{1}{p(s)} \int_{0}^{s} p(x) q(x) g(a-\varphi(x)) d x d s & \leq a-\varphi(t), \\
\int_{0}^{t} \frac{1}{p(s)} \int_{0}^{s} p(x) q(x) g(\varphi(x)) d x d s & \geq \varphi(t)
\end{aligned}
$$

for some $\varphi(t) \in C[0, T]$, with $0 \leq \varphi(t) \leq a$. Then (1.1) has a solution $y \in C[0, T]$ with $p y^{\prime} \in C[0, T],\left(p y^{\prime}\right)^{\prime} \in L_{p q}^{1}(0, T)$ and $0<y(t) \leq$ a for $t \in[0, T]$. In addition if either

$$
p(0) \neq 0
$$

or

$$
p(0)=0 \text { and } \lim _{t \rightarrow 0+} \frac{p(t) q(t)}{p^{\prime}(t)}=0
$$

holds, then $y$ is a solution of (1.2).

Remark 2.2. The case $\varphi(t) \equiv 0$, corresponds to the case of inequality

$$
\int_{0}^{t^{*}} \frac{1}{p(s)} \int_{0}^{s} p(x) q(x) g(a) d x d s<\text { a for any } t^{*} \in(0, T] .
$$

Proof of Theorem 2.1. Consider the sequence $\left\{y_{n}(t)\right\}, n=0,1,2, \ldots$ with $y_{0}(t) \equiv a-\varphi(t)$,

$$
y_{n}(t)=a-\int_{0}^{t} \frac{1}{p(s)} \int_{0}^{s} p(x) q(x) g\left(y_{n-1}(x)\right) d x d s, n=1,2, \ldots, t \leq T .
$$

We have

$$
\begin{aligned}
y_{0}(t) & \equiv a-\varphi(t) \\
y_{1}(t) & =a-\int_{0}^{t} \frac{1}{p(s)} \int_{0}^{s} p(x) q(x) g(a-\varphi(x)) d x d s \geq \varphi(t), \\
y_{2}(t) & =a-\int_{0}^{t} \frac{1}{p(s)} \int_{0}^{s} p(x) q(x) g\left(y_{1}(x)\right) d x d s \\
& \leq a-\int_{0}^{t} \frac{1}{p(s)} \int_{0}^{s} p(x) q(x) g(\varphi(x)) d x d s \\
& \leq a-\varphi(t),
\end{aligned}
$$




$$
\begin{aligned}
y_{3}(t) & =a-\int_{0}^{t} \frac{1}{p(s)} \int_{0}^{s} p(x) q(x) g\left(y_{2}(x)\right) d x d s \geq \varphi(t) \\
y_{4}(t) & =a-\int_{0}^{t} \frac{1}{p(s)} \int_{0}^{s} p(x) q(x) g\left(y_{3}(x)\right) d x d s \leq a-\varphi(t), \ldots \\
y_{2 n-1}(t) & \geq \varphi(t), \\
y_{2 n}(t) & \leq a-\varphi(t), \ldots
\end{aligned}
$$

The sequences $\left\{y_{2 n}(t)\right\}$ and $\left\{y_{2 n+1}(t)\right\}$ are equicontinuous. Indeed we have

$$
\left|y_{n}(t)-y_{n}(r)\right|=\int_{r}^{t} \frac{1}{p(s)} \int_{0}^{s} p(x) q(x) g\left(y_{n-1}(x)\right) d x d s \leq M \int_{r}^{t} \frac{1}{p(s)} \int_{0}^{s} p(x) q(x) d x d s,
$$

where

$$
M=\max \{g(u): 0 \leq u \leq a\}
$$

and the right hand side of (2.1) can be taken $<\varepsilon$ for $|t-r|<\delta$, regardless of the choice of $t$ and $r$ : the function $\int_{0}^{t} \frac{1}{p(s)} \int_{0}^{s} p(x) q(x) d x d s$ is (uniformly) continuous on $[0, T]$. It follows from Ascoli Arzela Theorem that the sequence $\left\{y_{2 n}(t)\right\}$ has the (uniformly) convergent subsequence, $y_{2 n_{k}}(t) \rightarrow u(t)$. The Lebesgue dominated theorem guarantees that

$$
\begin{aligned}
y_{2 n_{k}+1}(t) & =a-\int_{0}^{t} \frac{1}{p(s)} \int_{0}^{s} p(x) q(x) g\left(y_{2 n_{k}}(x)\right) d x d s \rightarrow v(t), \\
v(t) & =a-\int_{0}^{t} \frac{1}{p(s)} \int_{0}^{s} p(x) q(x) g(u(x)) d x d s \\
\text { and } u(t) & =a-\int_{0}^{t} \frac{1}{p(s)} \int_{0}^{s} p(x) q(x) g(v(x)) d x d s .
\end{aligned}
$$

If $u(t)=v(t)$ we have that the function $u(t)$ is the solution of the problem (1.1), indeed it follows from

$$
u(t)=a-\int_{0}^{t} \frac{1}{p(s)} \int_{0}^{s} p(x) q(x) g(u(x)) d x d s
$$

that

$$
\begin{aligned}
u^{\prime}(t) & =-\frac{1}{p(t)} \int_{0}^{t} p(x) q(x) g(u(x)) d x, \\
p u^{\prime} & =-\int_{0}^{t} p(x) q(x) g(u(x)) d x, \\
\left(p u^{\prime}\right)^{\prime} & =-p q g(u) .
\end{aligned}
$$

So we suppose $u(t) \neq v(t)$. We have $u(0)=v(0)=a$ and if for example, $u(t)>v(t)$ on the interval $(0, b)$, then we obtain

$$
u(b)-v(b)=\int_{0}^{b} \frac{1}{p(s)} \int_{0}^{s} p(x) q(x)[g(u(x))-g(v(x)] d x d s>0
$$

and therefore $u(t)>v(t)$ on the whole interval $(0, T]$. The same holds for all points of intersections $t_{0}: u\left(t_{0}\right)=v\left(t_{0}\right)$. That is if $u\left(t_{0}\right)=v\left(t_{0}\right)$, then for any $\varepsilon>0$ there are infinitely many points $t_{n} \in\left[t_{0}, t_{0}+\varepsilon\right)$ such that $u\left(t_{n}\right)=v\left(t_{n}\right)$. Therefore, $u(t)>v(t)$ (or $<$ ) on $\left(t_{0}, T\right]$. Without loss of generality let us suppose $u(t)>v(t)$ on $(0, T]$

and consider the operator $N: C[0, T] \rightarrow C[0, T]$ defined by

$$
N y(t)=a-\int_{0}^{t} \frac{1}{p(s)} \int_{0}^{s} p(x) q(x) g(y(x)) d x d s .
$$

Next let 


$$
K=\{y \in C[0, T]: v(t) \leq y(t) \leq u(t) \text { for } t \in[0, T]\}
$$

Clearly $K$ is closed, convex, bounded subset of $C[0, T]$ and $N: K \rightarrow K$. Let us show that $N: K \rightarrow K$ is continuous and compact operator. Continuity follows from Lebesgue dominated convergence theorem: if $y_{n}(t) \rightarrow y(t)$, then $N y_{n}(t) \rightarrow N y(t)$. To show that $N$ is completely continuous let $y(t) \in K$, then

$$
|N y(t)-N y(r)| \leq M\left|\int_{r}^{t} \frac{1}{p(x)} \int_{0}^{x} p(z) q(z) d z d s\right| \text { for } t, r \in[0, T],
$$

that is $N$ completely continuous on $[0, T]$.

The Schauder-Tychonoff theorem guarantees that $N$ has a fixed point $w \in K$, i.e. $w$ is a solution of (1.1). It follows from

$$
w^{\prime}(t)=-\frac{1}{p(t)} \int_{0}^{t} p(x) q(x) g(w(x)) d x
$$

that if $p(0) \neq 0$ then $w^{\prime}(0)=0$. Now if $p(0)=0$ but $\lim _{t \rightarrow 0+} \frac{p(t) q(t)}{p^{\prime}(t)}=0$ we have from (??) that

$$
w^{\prime}(0+)=-\lim _{t \rightarrow 0+} \int_{0}^{t} \frac{p(x) q(x)}{p(t)} g(w(x)) d x=0,
$$

that is $w$ is a solution of (1.2).

The proof is completed.

\section{Conflict of interests}

The author declares that there is no conflict of interests regarding the publication of this paper.

\section{References}

[1] R. P. Agarwal, D. O’Regan, Second order initial value problems of Lane-Emden type, Appl. Math. Lett., 20(12) (2007), 1198-1205.

[2] A. Aslanov, A note on the existence of positive solutions of singular initial-value problem for second order differential equations, Electron. J. Qual. Theory Differ. Equ., 84 (2015), 1-10. 